\title{
Correction of residual metatarsus adductus deformity following ponseti management of idiopathic clubfoot in toddlers by tibialis anterior tendon transfer
}

\begin{abstract}
Background: Ponseti technique becomes a gold standard treatment for correction of idiopathic clubfoot and widely reports to provide reliable results. However, a relapsed deformity may occur owing to imbalance of strong tibialis anterior and weak its antagonist resulting in dynamic metatarsus adductus deformity of forefoot. The aim of this study is reporting the results of our experience regarding tibialis anterior tendon transfer for correction of previous deformity.
\end{abstract}

Patients and methods: In this prospective case series study, 21 child (9 females) with residual dynamic metatarsus adductus following treatment of idiopathic congenital talipes equinovarus utilizing Ponseti technique treated by transfer of tibialis anterior tendon into third cuneiform bone. Evaluation comprised clinical, appearance, radiological assesment and complications.

Results: Mean follow-up 23.4 months and mean age of patients 35.3months. All transferred tendons healed properly without major complications. Clinically; Dimeglio Score improved from 5.8 preoperative to 1.3 postoperative and the clinical appearance according to Garceau and Palmer improved from 2.8 to 3.4. No major complications or correction loss developed in any case.

Conclusion: Residual dynamic metatarsus adductus deformity following Ponseti management of congenital talipes equinovarus can be corrected efficiently and simply by tibialis anterior tendon transfer.

Keywords: Idiopathic Clubfoot, Tibialis anterior tendon, Metatarsus adductus, Ponseti
Volume 9 Issue I - 2017

Mohamed Abdel-AAI

El-Bakry General Hospital, Egypt

Correspondence: Mohamed Abdel-AAI, El-Bakry General Hospital, 6I Taha Heussein-Nozha Cairo, Egypt, Tel 1020995024, Emailbtmnail2010@hotmail.com

Received: July 07, 2017 | Published: September 21, 2017

\section{Level of Evidence}

A. The author certifies that he has no commercial associations (eg, consultancies, stock ownership, equity interest, patent/licensing arrangements, etc) that might pose a conflict of interest in connection with the submitted article.

B. The author certifies that his institution has approved the human protocol for this investigation and that all investigations were conducted in conformity with ethical principles of research, and that informed consent for participation in the study had been obtained. Date of approval January/2013 and registration ID 02416.

\section{Introduction}

Residual metatarsus adductus is one of the common sequelae that may develop following correction of congenital club foot in children utilizing Ponseti technique. ${ }^{1}$ Its incidence varies in different studies and may reach up to $23 \%{ }^{2}$ Variable causes of relapsing of this particular deformity may be accused involving: inadequate correction of the original deformity (although the deformity has been reported despite good initial correction), muscle imbalance (from a strong supinatory action of tibialis anterior muscle and weak antagonists) or loss of reduction. ${ }^{3}$ Transfer of the anterior tibialis tendon is an effective method for correcting this deformity in children. It was originally described by Garceau ${ }^{4}$ and later modified by Ponseti \& Smoley. ${ }^{5}$ Current treatment methods describe either whole tendon subcutaneously beneath or above the ankle retinaculum or split tendon transfer with pros. and cons. for each method. ${ }^{6-8}$ At our institution we have used the technique of transfering the whole tibialis anterior tendon subcutaneously to the 3rd cuniform bone according to Ponseti \& Smoley ${ }^{5}$ Technique, thereby maintaining its position beneath the ankle retinaculum.

\section{Patients and methods}

Our prospective case series study was initiated after receiving approval from the institutional ethics committee for research in accordance with the ethical standards laid down in the 1964 declaration of Helsinki and its later amendments. Also, a written consent had been obtained from the patient's parents or relatives for participating in the study. Between March 2013 and November 2015, 21 children (9females) with 37 feet and met the inclusion criteria had been enrolled in this study. All had residual metatarsal adductus following treatment of idiopathic club foot deformity utilizing Ponseti technique. 16 cases had bilateral affection ( 32 feet) and 5 cases had unilateral foot deformity. Mean age 37.5 months (range 37-59months). Inclusion criteria involved: Children above 2 years age, correctable metatarsus adductus deformity, and idiopathic club foot and previously treated by Ponseti technique. Exclusion criteria involved: children below 2 years, fixed non correctable deformity, associated deformity other than metatarsus adductus, patients treated surgically and deformity other than idiopathic club foot. Data were collected in a predesigned proforma which included detailed history obtained from the informants of the patient, developmental history, family 
history and history of conservative treatment and any complications developed. On clinical examination, the foot was examined to accurately realize the deformity present and the flexibility of foot was also examined based on the maximum possible correction achieved by manipulation. The follow-up period ranged from 18-30 months. The mean preoperative Dimeglio ${ }^{9}$ score was 5.8 and the clinical appearance according to Garceau \& Palmer $^{6}$ was 2.7

\section{Surgical technique}

All patients underwent routine pre-operative investigations as required. Full clinical examination including a thorough neurovascular assessment carried out. Standard anteroposterior and lateral radiographs of the involved elbow taken. The patient was placed in supine position on a radiolucent table. A third generation Cephalosporin was administered before induction of anaesthesia. Tourniquet was inflated after exsanguinations the limb.

Two incisions were made, one from inferior margin of extensor retinaculum to distal insertion of the tendon aimed at detaching it from its insertion on the medial aspect of the foot (Figure 1). Care was taken to preserve as much length as possible by mobilizing the entire insertion of the tendon as there could be two or three slips at the insertion site. The tendon was dissected proximally to the ankle retinaculum and secured with a Bunnell-type suture using a large 0 or number 1 non absorbable suture. This maintained control of the tendon and prevented it from becoming bulbous during transfer (Figure 2). A second incison done over the third cuneiform and the soft tissues elevated to expose it. A hole drilled in the third cuneiform from dorsal to plantar and the opening of the hole was widened slightly with a large curette (Figure 3). This hole was the same diameter as the tendon. A subcutaneous tunnel was created between the two incisions keeping the transferred tendon underneath the extensor retinaculum of the ankle to prevent bowstringing. Finally the tendon passed through the drilled hole and withdrawn from the plantar surface of foot and sutured tightly under tension outside the skin over pieces of rubber tubing while the foot placed in the corrected position of abduction (Figure 4). Also, the tendon sutured to the periosteum and additional sutures taken between the soft tissues around the whole entrance and the tendon to improve the stability of fixation. Proper haemostasis done following deflation of tourniquet and the wounds closed. An above knee cast with knee flexed $90^{\circ}$ applied and the foot maximally abducted (Figure 5).

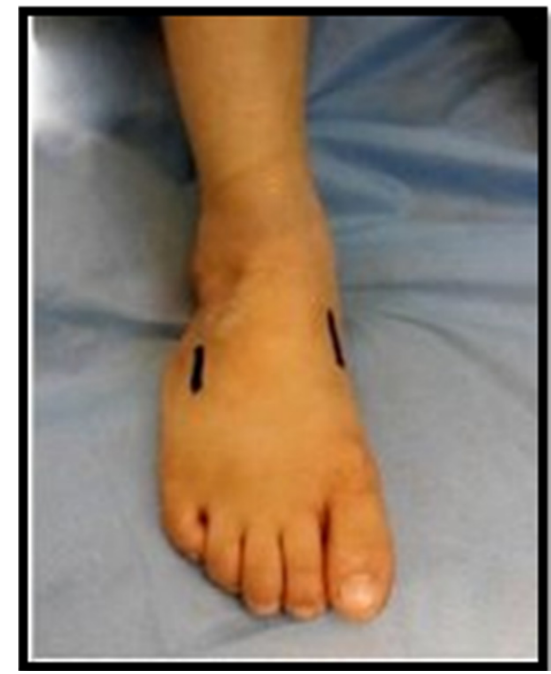

Figure I Two incisions, one medial from inferior margin of extensor retinaculum to distal insertion of tendon. A second incison lateral over third cuneiform.

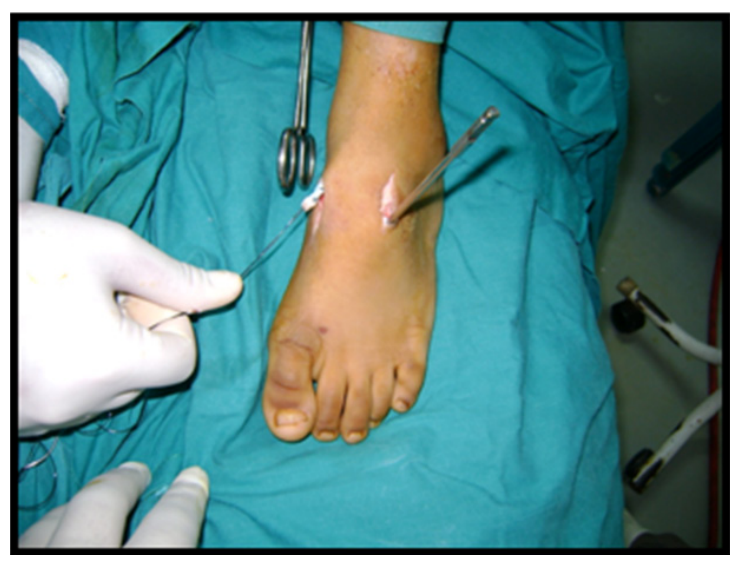

Figure $\mathbf{2}$ The tendon dissected proximally to the ankle retinaculum and secured with a Bunnell-type suture.

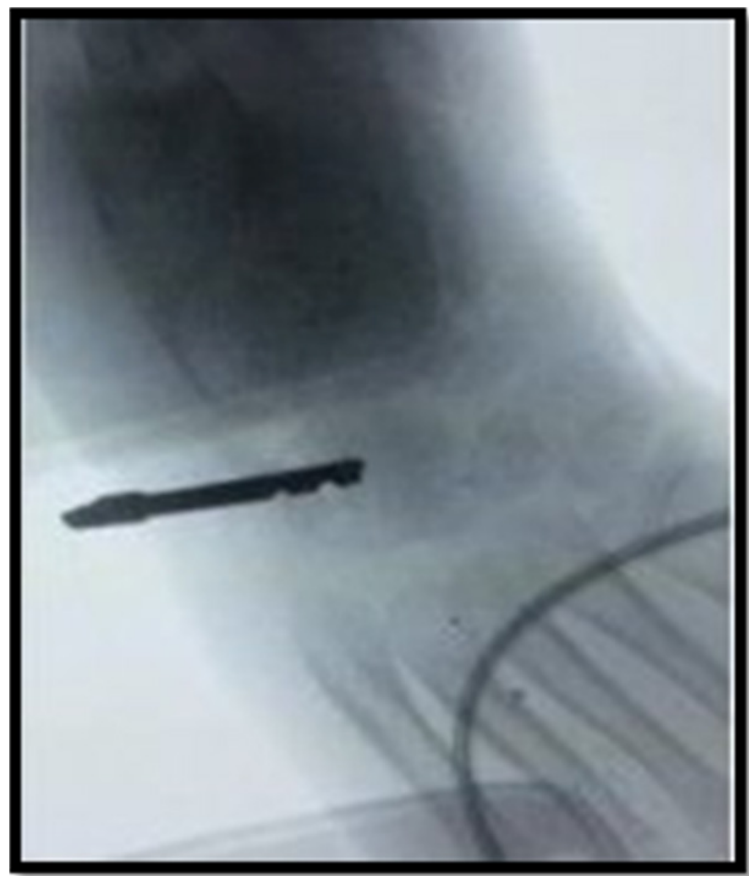

Figure $3 \mathrm{~A}$ hole drilled in the third cuneiform from dorsal to plantar.

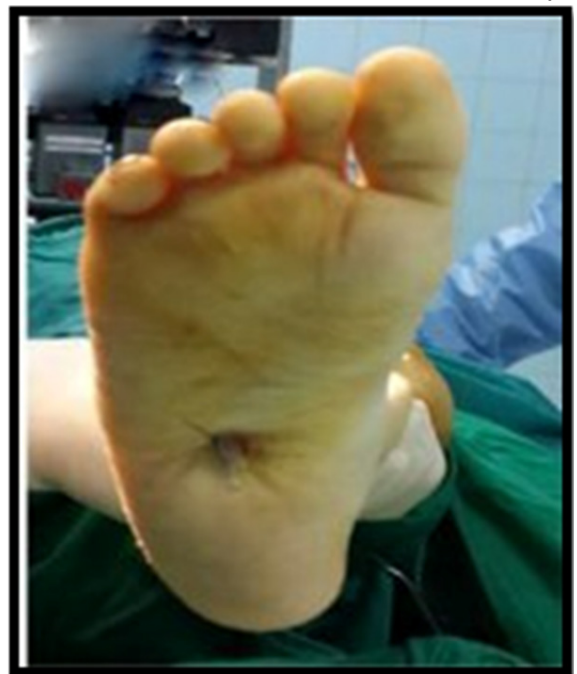

Figure 4 The tendon passed through the drilled hole and withdrawn from the plantar surface of foot. 


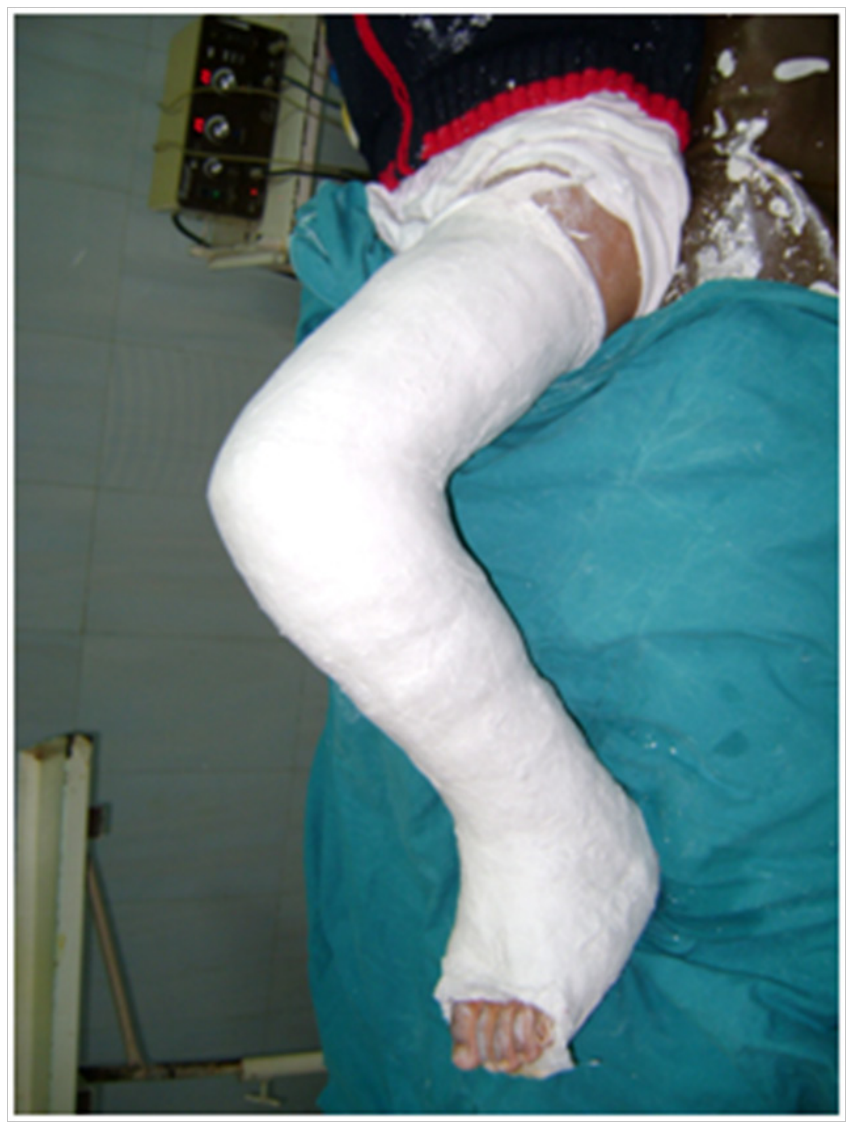

Figure 5 Above knee cast with 90 degree knee flexion.

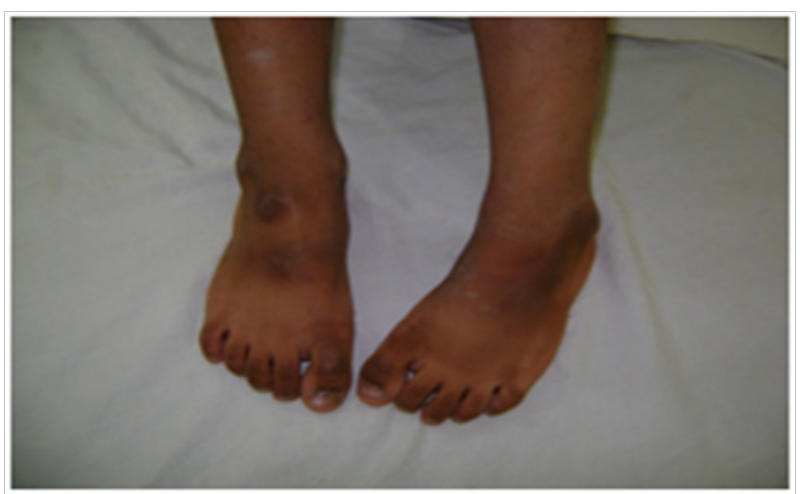

A. Child 5 years with residual left metatarsus adductus.

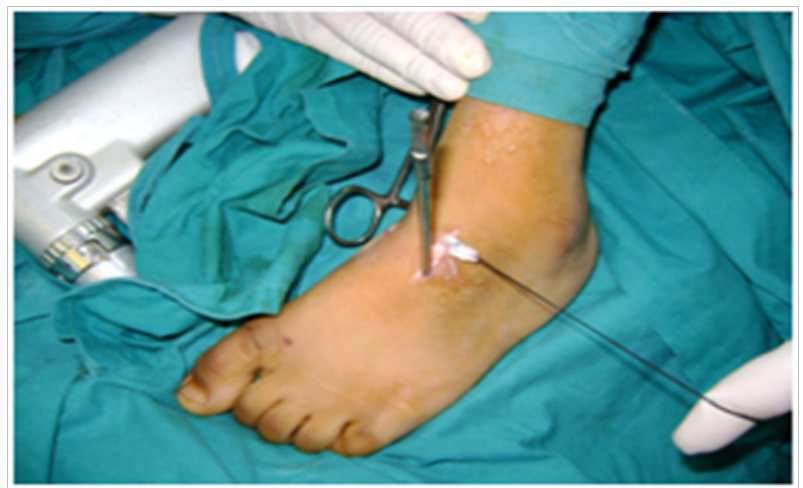

B. Intraoperative during tendon transfer

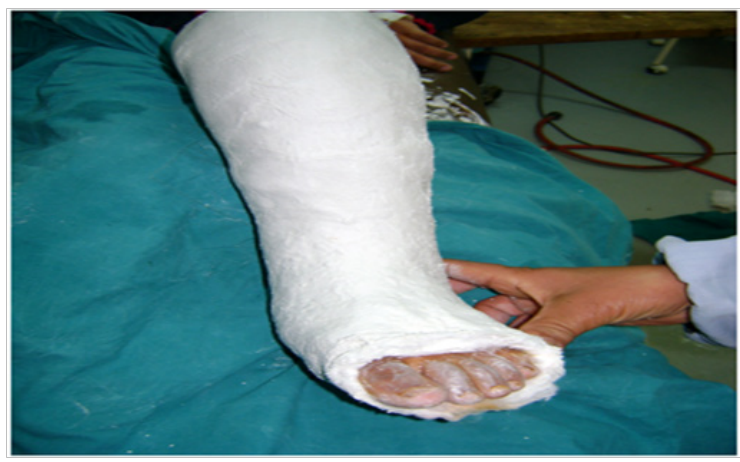

C. Above Knee cast with knee flexion and abduction of forefoot.

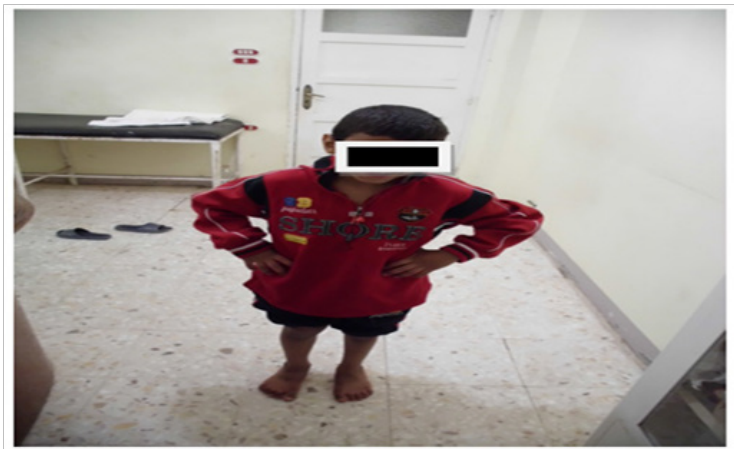

D. After cast removal anf full weight bearing One moth postoperative.

Figure 6 Case Example.

\section{Post operative regimen}

Cast maintained for 4 weeks then sutures were removed and weight bearing short-leg cast applied for more 3 weeks. Antibiotic continued to all patients for 72 hours. All Patients discharged after postoperative day three. All cases advised to attended outpatient clinic every week for one month then continued at 3,6 and 12 months for 2 years. The mean follow-up was 23.4 months (range 18-33months). The patients were evaluated clinically through Dimeglio Score, ${ }^{9}$ clinical appearance according to Garceau and Palmer. ${ }^{5}$ Radiographic assessment was done at last follow-up visit. Standing Antero-posterior radiographs of both feet were taken and naviculer-first cuneiform angle measured (indicator to amount of displacement of cuneiform. Also; calcanealfifth metatarsal angle determined (indicator of hind foot-fore foot alignment). Decrease in values of two angles represented amount of forefoot abduction hence the amount of correction of metatarsus adductus.

\section{Results}

Between March 2013 and November 2015, 21 patients with 37 feet who satisfied the inclusion and exclusion criteria were enrolled in this study. Age range (37-59months) with mean 35.3 months. The mean follow-up 23.4 months (range; 18-30months) and the mean hospital stay 2.1 days (range 2-5 days). All transferred tendons healed properly. Statistical analysis was performed using the Wilcoxon signed-rank test. Mean preoperative Dimeglio score was 5.8 preoperatively which improved to 1.3 postoperatively. The clinical appearance of all feet was improved according to the criteria of Garceau and Palmer ${ }^{6}$ from a preoperative mean of 2.7 to a mean of 3.4 at the final follow up. Radiographic evaluation revealed an average preoperative naviculofirst cuneiform angle $-21.7^{\circ}$ (range $-30^{\circ}$ to $-17^{\circ}$ ) which had been improved to an average $-3.7^{\circ}$ (range $-15^{\circ}$ to $-7^{\circ}$ ). The average 
calcaneo-fifth metatarsal angle improved from an average $-8.4^{\circ}$ (range $-21^{\circ}$ to $-13^{\circ}$ ) preoperatively to an average $-3.1^{\circ}$ (range $-7^{\circ}$ to $0^{\circ}$ ). No major complications apart from 6 feet had wound healing problem. There was no loss of correction and none of the operated feet had any relapse.

\section{Discussion}

Residual metatarsus adductus deformity frequently occurs following Ponseti manipulation of Idiopathic club foot. Its incidence varies among different studies. ${ }^{2,10}$ Transfer of the anterior tibialis tendon to lateral aspect of the foot is an effective method for correcting this deformity in children and had been recommended by many authors. Variable technique and modifications had been proposed for this transfer. Garceau ${ }^{4}$ recommended the whole tendon transfer which later on modified by Ponseti \& Smoley. ${ }^{5}$ Split tendon transfer had been recommended later on with good results. ${ }^{11,12}$

In our study, 37 feet with residual metatarsus adductus are managed by whole tibialis anterior tendon transfer to third cuneiform bone. The advantages of the technique (being passed behind the ankle retinaculum) maintain the normal position of the tendon beneath the ankle retinaculum which preserves its normal mechanics and prevents the tendon from bowstringing when the foot is dorsiflexed. This phenomenon minimizes the motor strength of the tendon and can cause tendon irritation. Also, it is cosmetically acceptable without any irritation of the overlying skin as it rubs against the shoe..$^{13}$ In our series we perform surgical intervention at mean age of 35.3 months (about 3 years) before worsening of the deformity and the foot becomes stiff. Also, the operation should be delayed till radiographic appearance of ossific center of $3^{\text {rd }}$ cuneiform (appear at about 2 years age).

The improved Dimeglio score $^{9}$ and the clinical appearance according to the criteria of Garceau ${ }^{6}$ of all feet at the final follow up proves that tibialis anterior transfer effectively corrects residual metatarsus adductus deformity following Ponseti management for idiopathic congenital talipes equinovarus. Radiographic evaluation of our series shows a decrease of values of both naviculer-first cuneiform and calcaneus-fifth metatarsal angles from preoperative to final follow-up values. This decrease may be due to the abductioneversion force caused by the tendon transfer that is able to shift the cuneiform and the whole forefoot more laterally. Also; the values of previous angles correlated with the clinical appearance of foot as the more negative angles the better the clinical results. ${ }^{13,14}$

When comparing ourresults with those of other studies on tibialis anterior tendon transfer of either the entire tendon above or below the ankle retinaculum or the split transfer, there appear to be no marked clinical differences despite a variety of assessments.

Laaveg ${ }^{15}$ reported good clinical and functional results in a long term follow-up study utilizing the whole tendon transfer into 3rd cuneiform. The study has been reviewed 30 years later by Cooper and Dietz ${ }^{16}$ who recommend that transfer of anterior tibial tendon to third cuneiform is the most efficient procedure of correcting residual clubfoot deformity following Ponseti technique.

Kuo et al. ${ }^{7}$ reviewed 55 patients ( 71 clubfeet) who had residual dynamic deformities and underwent full and split tibialis anterior tendon transfer. Both transfers were brought anterior to the ankle retinaculum. Using Dimeglio score and the clinical appearance according to Garceau and Palmer criteria, preoperatively in the entire tendon transfer group the mean score was 2.6points postoperatively, this improved to a mean of 3.3 points. In the split transfer group the mean preoperative score was 2.9 points which improved postoperatively to score 3.4 points. These results are more or less similar to the results of our study. Kuo et al. ${ }^{7}$ concluded that both procedures were effective but preferred the split tibialis anterior tendon transfer as they believed it was less likely to result in over connection and that it preserved some inversion function.

The main limitations to our study include: small patient numbers so; larger trials will improve precision of the findings. The follow-up period is somewhat short which may affect the long term complications that might develop. All the cases are candidates of previous Ponseti technique which may be non-compliant to some patients' parents and this could not be objectively confirmed (Figure 6).

\section{Conclusion}

Tibialis anterior tendon transfer effectively corrects dynamic metatarsus adductus following Ponseti management for idiopathic congenital talipes equinovarus.

\section{Author contribution}

The first author contribute for surgical intervention and arrangement of data, while second author contribute for collecting review of literature and share in surgical issue.

\section{Conflicts of interest}

Authors declare there is no conflict of interest in publishing the article.

\section{Acknowledgements}

None.

\section{References}

1. Ponseti IV EN S. Congenital Club Foot: The Results of Treatment. $J$ Bone Joint Surg Am. 1963;45:261-344.

2. Abdelgawad AA, Lehman WB, van Bosse HJ, et al. Treatment of idiopathic clubfoot using the Ponseti method: minimum 2-year followup. J Pediatr Orthop. 2007;16:98-105.

3. Thompson GH, Hoyen H, Barthel T. Tibialis anterior tendon transfer after clubfoot surgery. Clin Orthop Relat Res. 2009;467(5):1306-1313.

4. Garceau GJ. Anterior tibial tendon transposition in recurrent congenital club-foot. J Bone Joint Surg Am. 1940;22:932-936.

5. Ponseti IV, Smoley EN. Congenital club foot: the results of treatment. $J$ Bone Joint Surg Am. 1963;45:261-275.

6. Garceau GJ, Palmer RM. Transfer of the anterior tibial tendon for recurrent club foot: a long-term follow-up. J Bone Joint Surg Am. 1967;49A:207-231.

7. Kuo KN, Hennigan SP, Hastings ME. Anterior Tibial Tendon Transfer in Residual Dynamic Clubfoot Deformity. J Pediatr Orthop. 2001;21(1):35-41.

8. Ippolito E, Ricciardi-Pollini PT, Tudisco C, et al. The treatment of relapsing clubfoot by tibialis anterior transfer underneath the extensor retinaculum. Ital J Orthop Traumatol. 1985;11(2):171-177.

9. Dimeglio A, Bensahel H, Souchet P, et al. Classification of clubfoot. $J$ Pediatr Orthop. 1995;4:129-136.

10. Dietz FR. Treatment of a recurrent clubfoot deformity after initial orrection with the Ponseti technique. Instr Course Lect. 2006;55:625-629.

11. MM H, JA R, AM GJP. The split anterior tibial tendon transfer in the treatment of spastic varus hindfoot of childhood. Orthop Clin North Am. 1974;(5):31-38. 
12. Hoffer MM, Reiswig JA, Garrett AM, et al. The split anterior tibial tendon transfer in the treatment of spastic varus hindfoot of childhood. Orthop Clin North Am. 1974;5(1):31-38.

13. Ezra E, Hayek S, Gilai AN, et al. Tibialis anterior tendon transfer for residual dynamic supination deformity in treated clubfeet. $J$ Pediatr Orthop. 2000;9(3):207-211.

14. Lampasi M, Bettuzzi C, Palmonari M, et al. Transfer of the Tendon of Tibialis Anterior in Relapsed Congenital Clubfoot: Long-Term Results in 38 Feet. J. Bone Joint Surg Br. 2010;92:277-283.
15. Laaveg SI, Ponseti IV. Long term results of treatment of congenital clubfoot. J Bone Joint Surg Am. 1980;62:23-31.

16. Cooper DM, Dietz FR. Treatment of idiopathic clubfoot. A thirty-year follow-up. J Bone Joint Surg Am. 1995;77(10):1477-1489. 